\title{
DETERMINAÇÃO DO GRADIENTE HIDRÁULICO MÉDIO EM AQUÍFERO COSTEIRO INFLUENCIADO PELA VARIAÇÃO \\ CONSTANTE DE CARGA HIDRÁULICA EM AMBIENTE ESTUARINO
}

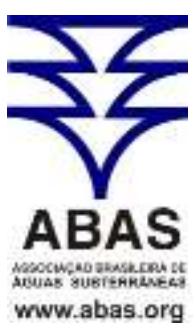

\author{
DETERMINATION OF MEAN HYDRAULIC GRADIENT IN COASTAL \\ AQUIFER UNDER INFLUENCE OF CONSTANT HEAD VARIATION IN \\ ESTUARINE ENVIRONMEN
}

\author{
Paulo Cesar Negrão ${ }^{1}$; Sueli Yoshinaga Pereira ${ }^{2}$; \\ Felipe Gasparini ${ }^{3}$; Valter Leite ${ }^{4}$
}

Artigo recebido em: 07/07/2015 e aceito para publicação em: 13/10/2016.

DOI: http://dx.doi.org/10.14295/ras.v30i3.28480

\begin{abstract}
Resumo: Na determinação dos gradientes hidráulicos das águas subterrâneas em aquíferos estuarinos, as ondas de pressão decorrentes das flutuações das marés e corpos de água devem ser consideradas. Para este estudo, monitorou-se por 45 dias as variações de nível de água do Rio Cubatão e carga hidráulica do aquífero raso (em 3 níveis de profundidade) numa área industrial adjacente ao rio, em Cubatão, SP, a fim de se determinar as cargas hidráulicas médias e gradientes médios pelo método conforme proposto por Serfes (1991). Este monitoramento foi contínuo e realizado em dois cenários: com o sistema de remediação ("Air Sparging") ativado e desativado. Os resultados mostraram a interferência do sistema de remediação e da variação da maré nas cargas hidráulicas dos poços monitorados nos poços próximos ao rio Cubatão, e somente a variação da maré nos poços mais distantes. No entanto, na porção superior do aquífero monitorado mostrou a condição de influência do rio Cubatão nos dois cenários. Condições de efluência e influência na porção intermediária e efluência predominante desse rio na porção mais profunda foram observadas. Nas porções intermediária e profunda, os gradientes hidráulicos calculados apresentam valores baixos $(-0,0027$ a 0,0030$)$, de modo que a análise sobre o sentido de fluxo deve ser criteriosa.
\end{abstract}

Palavras-chave: Água subterrânea. Ambiente estuarino. Gradientes hidráulicos. Cargas hidráulicas.

\begin{abstract}
In determining the hydraulic gradients of groundwater aquifers in estuarine environment, it is essential to consider the pressure waves resulting from tidal fluctuations and water bodies. The fluctuations of the water level of the Cubatão River and hydraulic head of the shallow aquifer (in 3 levels of depth) in Cubatão, SP, were monitored for 45 days in an adjacent industrial area to the river in order to determine the mean hydraulic head and mean hydraulic gradients by the method proposed by Serfes (1991). The monitoring was continuous and performed in two scenarios: with the remediation system ("Air sparging" technology) activated and deactivated. The results showed the interference of the remediation system and the variations of the tide in hydraulic heads of the nearby wells, and only the variation of the tide in the more distant wells. However, in the upper aquifer the influent condition of the Cubatão river was present in both scenarios. Effluent and influent conditions in the intermediate portion, and predominant effluent of the river in the deep portion were observed. In the intermediate and deep portions of the aquifer, the hydraulic gradients have low values $(-0.0027$ to 0.0030$)$ so that the analysis of the flow direction have to be careful.
\end{abstract}

Keywords: Groundwater. Estuarine environment. Hydraulic gradient. Hydraulic heads.

\section{INTRODUÇÃO E OBJETIVOS}

Aquíferos adjacentes a corpos d'água influenciados pela variação de nível das marés em ambiente estuarino sofrem variações constantes nas suas cargas hidráulicas em resposta à dinâmica destes sistemas aquáticos, decorrentes da variação das marés (WESTBROOK,

${ }^{1-4}$ Instituto de Geociências, Universidade Estadual de Campinas - E-mails: (pcnegrao@mac.com, sueliyoshinaga@gmail.com , felipe gasparini@hotmail.com, valter@fullgeo.com.br ) 
2005).

A determinação dos gradientes hidráulicos destes aquíferos representa um grande desafio para os profissionais de águas subterrâneas, uma vez que medições pontuais das cargas hidráulicas não levam em consideração as dinâmicas do ambiente estuarino em função da variação das marés. Serfes (1991), Robinson et al (1998), Ricardi-Branco et al (2011), Souza et al. (2012), Batista Filho (2006), Gasparini (2014), Abarca et al. (2013) são alguns autores que apresentaram estudos sobre a dinâmica das águas subterrâneas com a influência das marés, principalmente nas oscilações e variações do sentido de fluxo que ocorrem na superfície potenciométrica dos aquíferos adjacentes com a pressão exercida pelas marés.

O presente estudo avalia a aplicação da Metodologia de Médias Móveis proposta por Serfes (1991) para a determinação das cargas hidráulicas médias em aquíferos influenciados por variações de carga em ambiente estuarino e, por sua vez, os gradientes hidráulicos médios. Para tanto, foram monitoradas as variações de nível do Rio Cubatão, no município de Cubatão, SP, no período de 22 de no- vembro de 2012 a 03 de janeiro de 2013 e o monitoramento das cargas hidráulicas do aquífero local em 8 poços de monitoramento (em conjunto de 3 poços multiníveis) instalados em área industrial adjacente ao Rio Cubatão. Este estudo objetivou avaliar a aplicação do Método de Serfes (1991), com base nas informações de monitoramento de níveis de água subterrânea e do rio Cubatão.

\section{HIDROLOGIA E HIDROGEOLOGIA LOCAL}

Localizada na cidade de Cubatão no Estado de São Paulo (latitude 23 53' 44" S e longitude $46^{\circ} 25^{\prime} 32^{\prime \prime} \mathrm{O}$ ), próxima à escarpa da Serra do Mar, a área em estudo está localizada em um terreno sob propriedade de uma indústria petroquímica que produz matérias primas de produtos derivados de hidrocarbonetos aromáticos. A área de estudo faz divisa com o Rio Cubatão, o qual tem sua nascente no ponto geográfico de latitude $23^{\circ} 56^{\prime} 11^{\prime \prime} \mathrm{S}$ e longitude $46^{\circ} 57^{\prime} 20^{\prime \prime} \mathrm{O}$, e deságua no município de Santos por cursos de águas superficiais em meio ao mangue (Figura 1).

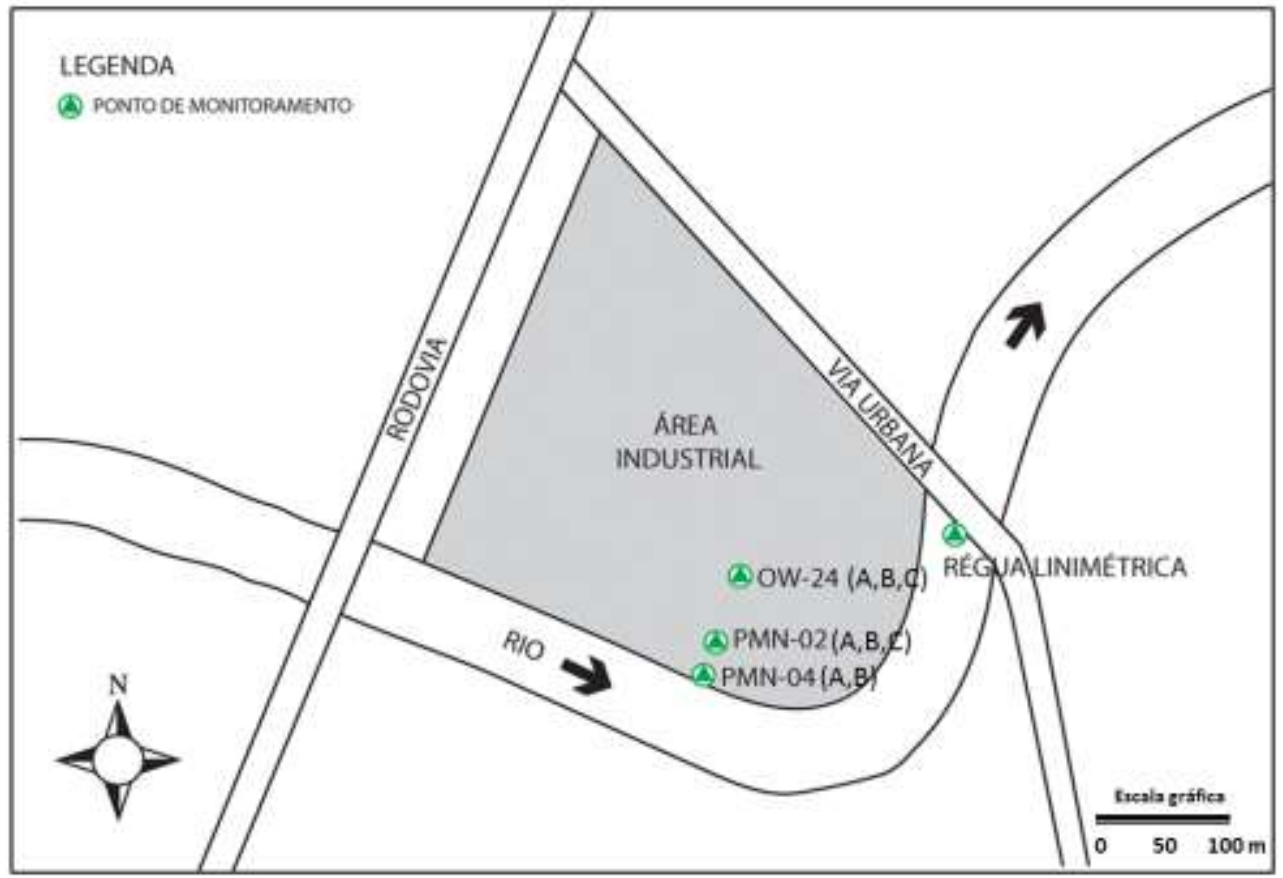

Figura 1 - Localização da área de estudo, distribuição espacial de poços de monitoramento multiníveis (PMN-02A, PMN-02B, PMN-02C, PMN-04A, PMN-04B, OW-24A, OW24B) e posição da régua linimétrica.

Figure 1 - Localization of the study area, spatial distribution of multilevel monitoring wells (PMN-02A, PMN-02B, PMN-02C, PMN-04A, PMN-04B, OW-24A, OW-24B) and limnimetric scale position. 
O aquífero do local de estudo é poroso, caracterizado por camadas predominantemente arenosas e de alta permeabilidade. Este domínio está representado por depósitos relacionados à idade Cenozóica, como sedimentos de aluviões, coluviões, depósitos eólicos, areias litorâneas, depósitos flúvio lagunares, arenitos de praia, deposito de leques-aluviais, depósitos de pântanos e mangues, coberturas detríticas e detríticas-lateríticas diversas e coberturas residuais, conforme contribui AECOM (2012).

$\mathrm{O}$ aquífero na área de monitoramento é adjacente ao Rio Cubatão, o qual faz parte do sistema estuarino da Baixada Santista e está conectado ao Oceano Atlântico através de um complexo deltaico de canais e manguezais (Cubatão, Prefeitura Municipal, http://www.Cubatão.sp.gov.br/historia/cidade/aspectos-geograficos/, acesso 15 março de 2015).

$\mathrm{Na}$ área industrial foi monitorado um aquífero de caráter livre em três camadas de diferentes profundidades, a saber (AECOM, 2012):

- Camada 1 (Raso): representa o topo do aquífero onde ocorre predominantemente sedimentos arenosiltosos representativos do aterro. Essa camada compreende o intervalo entre a franja capilar e o início do nível intermediário (Camada 2), em média, a 3,1 m de profundidade.

- Camada 2 (Intermediário): ocorre predominantemente sedimentos arenosos de granulação fina, com presença de cascalhos, representativos do Aluvião do rio Cubatão. Esse nível está compreendido entre as profundidades médias de $3,1 \mathrm{~m} \mathrm{e}$ $6,0 \mathrm{~m}$.

- Camada 3 (profundo): situa-se no intervalo entre 6,0 a 9,0 $\mathrm{m}$ de profundidade a partir da superfície do terreno. Este nível é composto por areia de granulação média a grossa representativa do Aluvião. É limitado na sua porção inferior por uma camada de argila marinha.

\section{MATERIAIS E MÉTODOS}

Este estudo contemplou o monitoramento contínuo das variações do nível d'água no Rio Cubatão, e do aquífero livre nos poços de monitoramento PMN-02A, PMN-02B, PMN-02C, PMN-04A, PMN-04B, OW-24A, OW-24B e OW-24C, com frequência de coleta de dados dos níveis potenciométricos dos corpos hídricos superficial e subterrâneo de 10 minutos. Os valores das cotas topográficas dos poços de monitoramento foram obtidos por meio de levantamentos planialtimétricos, com o auxílio de um nivelador óptico da marca CST/Berger ${ }^{\circledR}$ do modelo SAL28ND ${ }^{\circledR} \mathrm{e}$ régua métrica graduada, a partir de visadas na mira, sobrepostas à superfície do terreno. Os levantamentos foram realizados nos mesmos pontos em que são coletados os dados de níveis d'água dos poços de monitoramento e referenciados com base na régua de nível da maré instalada na base da ponte da Avenida Nove de Abril (Cubatão / SP).

A campanha de monitoramento teve início em 22 de novembro de 2013 e encerramento em 03 de janeiro de 2013. Para tanto, foram instalados transdutores de pressão em todos os pontos de monitoramento (rio Cubatão e poços de monitoramento) e um barômetro. Os relógios internos de todos os instrumentos foram sincronizados com um computador portátil, de maneira que todas as leituras ocorressem simultaneamente em todos os transdutores. O barômetro registrou as variações da pressão barométrica na microrregião do estudo em intervalos de 1 hora, a fim de que fossem desconsideradas das leituras de cada instrumento a pressão atmosférica e suas variações durante as campanhas de monitoramento.

Após a campanha, os dados armazenados nos transdutores de pressão foram transferidos para um computador e processados quanto a compensação das variações barométricas pelos programas WinSitu (https://insitu.com/support/documents/win-situ-5-software/ acesso 22 de maio de 2016), da empresa In-Situ Inc e Diver-Office da empresa Schlumberger. A série de dados foi então 
compilada em planilhas eletrônicas em Microsoft Excel@, para o cálculo das cargas hidráulicas do Rio Cubatão e do aquífero, bem como dos gradientes hidráulicos.

Serfes (1991) discorre sobre as dinâmicas de variação de nível de aquíferos costeiros influenciados por flutuações estuarinas. Corpos hídricos superficiais respondem às variações das marés com flutuações senoidais de suas cargas hidráulicas. Por sua vez, a carga hidráulica de aquíferos conectados hidraulicamente a tais corpos d'água superficiais também são influenciados em função das variações da maré.

O aumento de carga hidráulica dos aquíferos em função das variações de nível em corpos d'água superficiais em ambiente estuarino dá-se em função da onda de pressão que é transmitida ao aquífero. Em aquíferos livres, a onda de pressão é produzida por mudanças no armazenamento em resposta ao esvaziamento e preenchimento dos espaços porosos (SERFES, 1991). De acordo com esse autor, a dinâmica das elevações de nível da água subterrânea bem como a magnitude e direção do gradiente hidráulico, sob influência das marés, variam sobre a média do gradiente hidráulico regional. Serfes apresenta que métodos de filtragem tradicionalmente utilizados para determinação do nível médio diário do mar pode ser aplicado as águas subterrâneas.

Westbrook et al. (2005) afirma que as interações entre aquíferos e corpos d'água superficiais em ambientes estuarinos são influenciados por vários processos, resultando em sistemas complexos sob o ponto de vista espacial e temporal. Os contrastes de densidade entre a água, normalmente doce dos aquíferos, e a água tipicamente salobra dos ambientes estuarinos e costeiros promovem a mistura e a circulação convectiva na zona de contato do aquífero com os corpos hídricos superficiais estuarinos, causando a intrusão de água salobra para dentro dos aquíferos costeiros adjacentes.

Serfes (1991) contribuiu para a determinação do gradiente hidráulico médio de aquíferos influenciados por flutuações estuarinas. A carga hidráulica e os gradientes hidráulicos em tais aquíferos variam constante- mente, conforme identificado na área de estudo. Em função desta constante variação, as cargas hidráulicas e gradientes hidráulicos não podem ser determinados em medições pontuais de campo como se faz em aquíferos regidos apenas pelos gradientes regionais e sazonais.

Consequentemente o autor propõe o monitoramento das cargas hidráulicas em intervalos de 60 minutos, por um período continuo de 71 horas, para que se determine o que chama de "nível médio" e "gradiente médio".

O método proposto por Serfes (1991) baseia-se na técnica estatística das Médias Móveis. Segundo o autor:

Filtros são utilizados para remover de
maneira eficiente os harmônicos lunares
e solares diurnos e semi-diurnos de uma
sequência de 71 ou 72 leituras consecuti-
vas de hora em hora dos níveis d'água. A
utilização de médias móveis permite a
obtenção de um nível médio filtrado para
o tempo médio das 72 horas. Primeira-
mente, uma sequência de médias é calcu-
lada para as primeiras 24 leituras, inici-
ando pela observação para a primeira
média e observação 48 para a última, re-
sultando num total de 48 médias. Em se-
guida uma série similar de médias é cal-
culada para 24 das médias da primeira
etapa do cálculo, resultando em 25 mé-
dias. Por último, calcula-se a média das
25 médias, resultando no nível médio na
$36^{a}$ hora.

A expressão matemática deste método pode ser notada como:

$\mathrm{O}_{1}, \mathrm{O}_{2}, \mathrm{O}_{3}, \ldots ., \mathrm{O}_{71}$ formam a série de 71 leituras no nível d'agua em intervalos de 1 hora é dada por:

A primeira sequência de médias $\left(\mathrm{X}_{\mathrm{i}}\right)$

$$
X_{i}=\sum_{K=0}^{23} \frac{O(K+i)}{24}
$$

onde $\mathrm{i}=1,2,3,4, \ldots ., 48$; dada por:

A primeira sequência de médias $\left(\mathrm{Y}_{\mathrm{i}}\right)$ é

$$
Y_{j}=\sum_{i=0}^{23} \frac{(X i+j)}{24}
$$

onde $\mathrm{j}=1,2,3,4, \ldots ., 25$; 

por:

E o Nível Médio (M) na hora 36 é dado

$$
M=\sum_{j=1}^{25} \frac{Y j}{25}
$$

(Equação 1)

No presente estudo, o período de monitoramento das cargas hidráulicas foi realizado em dois cenários distintos:

- Cenário (A), no qual o aquífero respondeu às flutuações diárias de nível do Rio Cubatão em função das marés enchentes e vazantes e também aos ciclos de partida e parada de um sistema de remediação por injeção de ar (AS) e extração de vapores (SVE) existente na área industrial, cujo sistema foi instalado em poços específicos alinhados paralelamente à margem do rio, do rio Cubatão e próximos aos conjuntos de poços monitorados PMN-04 e PMN02 (porção sul da área de estudo). Esta condição pôde ser observada do início do monitoramento no dia 22 de novembro de 2012 até por volta das 15:30 do dia 14 de dezembro de 2012.
- Cenário (B), no qual o aquífero respondeu somente às flutuações diárias de nível do Rio Cubatão em função das marés enchentes e vazantes. Este regime foi observado a partir do desligamento do sistema de remediação para manutenção preventiva até o final do monitoramento (03/01/2013).

\section{RESULTADOS E DISCUSSÕES}

\subsection{Monitoramento das variações de carga hidráulica e gradiente hidráulico}

O monitoramento das variações das cargas hidráulicas no rio e nos poços de monitoramento da área de estudo durante a campanha de monitoramento de 22/11/2012 a 03/01/2013 permite observar que o aquífero nos poços de monitoramento PMN-04A e PMN-04B, PMN-02A, PMN-02B, PMN-02C, e OW-24A e OW-24B responde diretamente $\mathrm{e}$ instantaneamente às flutuações de nível do Rio Cubatão, conforme ilustrado nas figuras 2, 3 e 4.

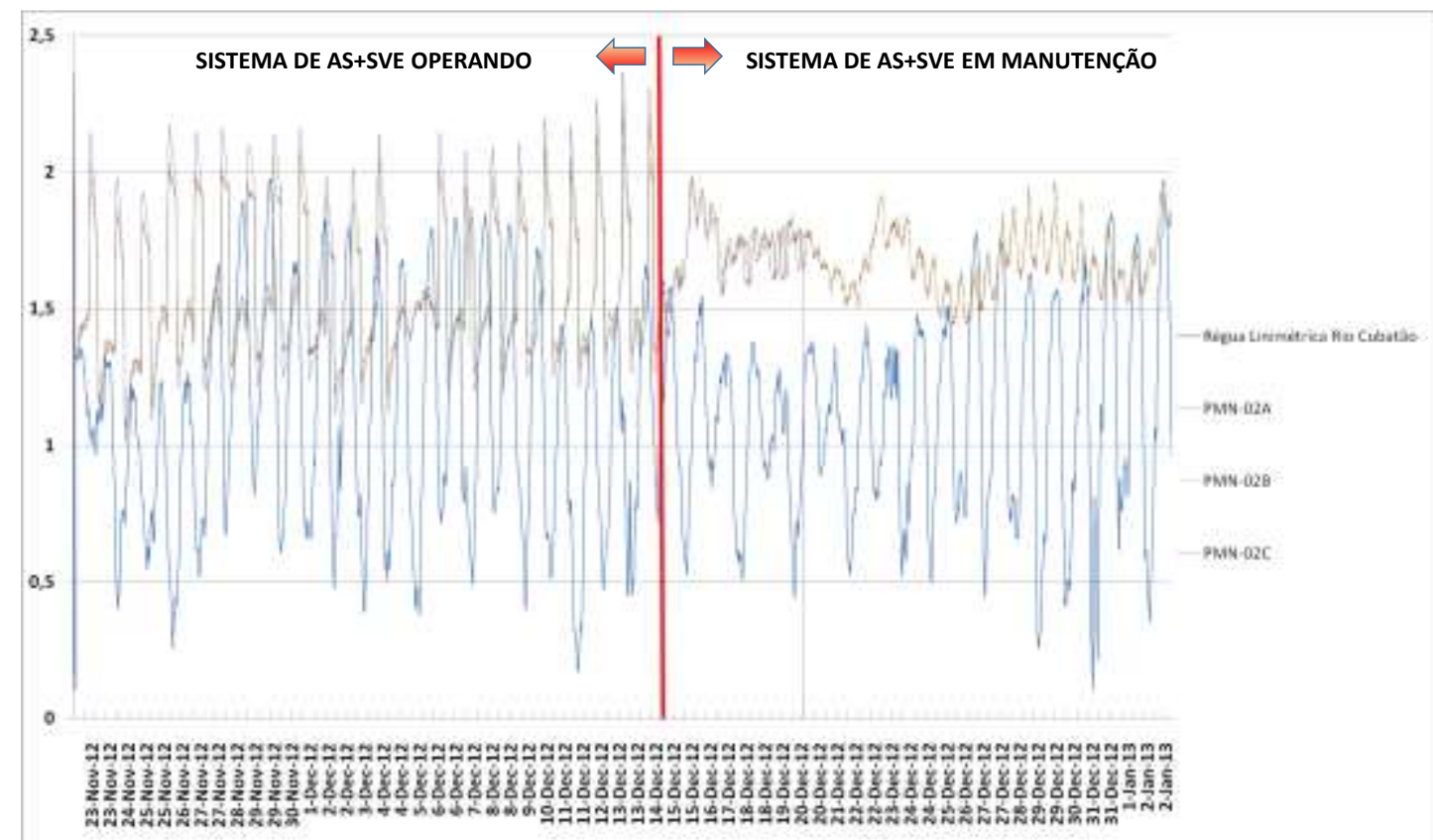

Figura 2 - Variações de nível d'agua no Rio Cubatão e nos poços de monitoramento PMN-02A, PMN-02B, PMN-02C (22/11 a 03/01/2013)

Figure 2 - Water fluctuations of Cubatão river and monitoring wells PMN-02A, PMN-02B, PMN-02C (Nov $22^{\text {nd }} 2012$ to Jan $3^{\text {rd }} 2013$ ) 


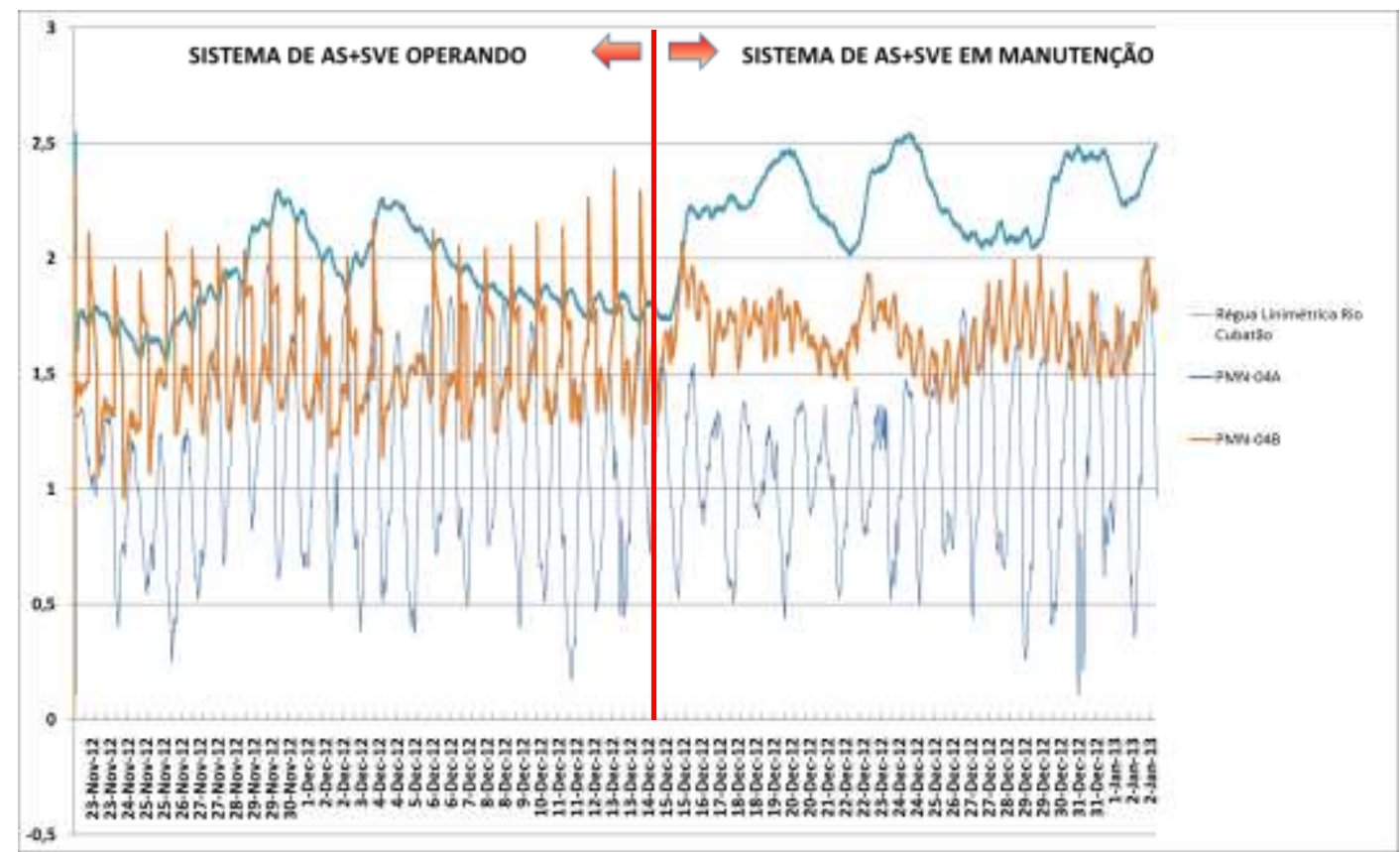

Figura 3 - Variações de nível d'agua no Rio Cubatão e nos poços de monitoramento PMN-04A e PMN04B (22/11 a 03/01/2013).

Figure 3 - Water fluctuations of Cubatão river and monitoring wells PMN-04A e PMN-04B (Nov 22 ${ }^{\text {nd }}$ 2012 to Jan $3^{\text {rd } 2013)}$

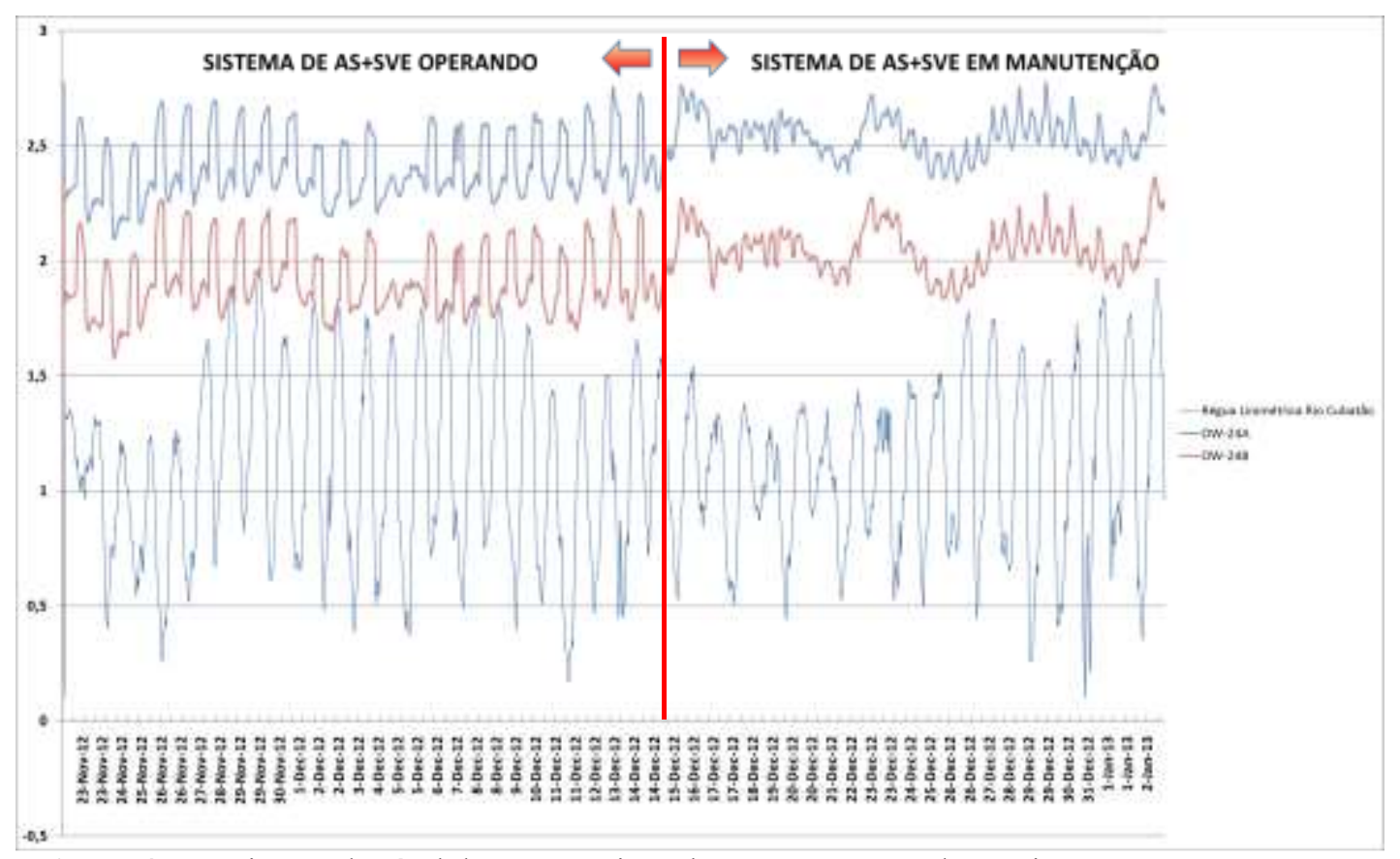

Figura 4 - Variações de nível d'agua no Rio Cubatão e nos poços de monitoramento OW-24A e OW-24B $(22 / 11 / 2012$ a $03 / 01 / 2013)$

Figure 4 - Water fluctuations of Cubatão river and monitoring wells OW-24A e OW-24B (Nov $22^{\text {nd }} 2012$ to Jan $3^{\text {rd } 2013)}$

Os gráficos apresentados nas figuras 2 e 3 apresentam a influência da operação do sistema de remediação na potenciometria do conjunto de poços multiníveis $\mathrm{PMN}-02$ e PMN-04. No Cenário A, quando o sistema de remediação operou em regime de 8 horas de operação por 18 horas em repouso, verifica-se o incremento das cargas hidráulicas dos poços PMN-02A, PMN-02B, PMN-02C, PMN-04A e PMN-04B da ordem de 0,60 a 0,70 m de co- 
luna d'água imediatamente após o acionamento do sistema de remediação. Esta constatação indica que esses poços estão na zona de influência do sistema de injeção de ar e extração de vapores. As cargas hidráulicas destes poços de monitoramento sofrem um decréscimo instantâneo de carga quando o sistema de remediação é desligado. Durante as 16 horas nas quais o sistema de remediação permanece em ciclo de repouso, o aquífero responde apenas às variações de carga do Rio Cubatão.

$\mathrm{Na}$ Figura 4 o par de poços OW-24, distantes a mais de $40 \mathrm{~m}$ dos poços $\mathrm{PMN}-02$, não sofrem influência das pressões introduzidas pelo sistema de remediação, porém somente as oscilações do rio são verificadas.

É possível notar que a partir das 15:30 do dia 14 de dezembro de 2012, com a parada para manutenção preventiva do sistema de remediação, o aquífero passa a responder somente às variações de carga do Rio Cubatão (figuras 3 e 4). Corroborando com Gasparini (2014), constata-se uma mudança brusca e significativa no regime de oscilação das cargas hidráulicas do aquífero a partir da parada para manutenção do sistema de remediação.

As cargas hidráulicas máximas e mínimas dos poços de monitoramento sob influência das variações de carga do Rio Cubatão e operação do sistema de remediação são apresentados na Tabela 1. A Tabela 2 apresenta as cargas máximas e mínimas do aquífero quando sujeito somente às variações de nível do Rio Cubatão.

Tabela 1 - Cargas Hidráulicas Médias, Máximas e Mínimas durante a operação do sistema de remediação no período de 22/11/2012 a 14/12/2012

Table 1 - Mean, Maximum and Minimum hydraulic heads during the remediation system in the period from Nov $22^{\text {nd }}$ to Dec $14^{\text {th }} 2012$

\begin{tabular}{ccccccccc}
\hline Carga & Rio & PMN- & PMN- & PMN- & PMN- & PMN- & OW- & OW- \\
Hidráulica & Cubatão & 02A & 02B & 02C & 04A & 04B & 24A & 24B \\
\hline Máxima & 1,974 & 2,225 & 2,253 & 2,365 & 2,695 & 2,385 & 2,754 & 2,265 \\
$\quad(m)$ & 1,089 & 1,552 & 1,551 & 1,555 & 2,310 & 1,538 & 2,399 & 1,908 \\
$\begin{array}{c}\text { Média (m) } \\
\text { Mínima } \\
(\mathrm{m})\end{array}$ & 0,170 & 1,065 & 1,055 & 1,013 & 1,955 & 0,955 & 2,094 & 1,575 \\
\hline
\end{tabular}

Tabela 2 - Cargas Hidráulicas Médias, Máximas e Mínimas apenas sob influência do Rio Cubatão no período de 14/12/2012 a 03/01/2013

Table 2 - Mean, Maximum and Minimum hydraulic heads only under influence of Cubatão river in the period from Dec $14^{\text {th }} 2012$ to Jan $3^{\text {rd }} 2013$

\begin{tabular}{|c|c|c|c|c|c|c|c|c|}
\hline $\begin{array}{c}\text { Carga } \\
\text { Hidráulica }\end{array}$ & $\begin{array}{c}\text { Rio } \\
\text { Cubatão }\end{array}$ & $\begin{array}{c}\text { PMN- } \\
02 \mathrm{~A}\end{array}$ & $\begin{array}{c}\text { PMN- } \\
02 \mathrm{~B}\end{array}$ & $\begin{array}{c}\text { PMN- } \\
02 \mathrm{C}\end{array}$ & $\begin{array}{c}\text { PMN- } \\
04 \mathrm{~A}\end{array}$ & $\begin{array}{c}\text { PMN- } \\
04 \mathrm{~B}\end{array}$ & $\begin{array}{l}\text { OW- } \\
24 \mathrm{~A}\end{array}$ & $\begin{array}{l}\text { OW- } \\
24 \mathrm{~B}\end{array}$ \\
\hline $\begin{array}{l}\text { Máxima } \\
(\mathrm{m})\end{array}$ & 1,925 & 1,975 & 1,991 & 1,965 & 2,945 & 2,075 & 2,774 & 2,365 \\
\hline Média (m) & 1,097 & 1,683 & 1,697 & 1,711 & 2,649 & 1,679 & 2,540 & 2,049 \\
\hline $\begin{array}{l}\text { Mínima } \\
(\mathrm{m})\end{array}$ & 0,104 & 1,395 & 1,418 & 1,403 & 2,135 & 1,355 & 2,309 & 1,785 \\
\hline
\end{tabular}

Na Tabela 2 as variações máximas e mínimas de carga hidráulica refletem a interferência do sistema de remediação. Na comparação entre os valores das Tabelas 2 e 3, a variação da cota máxima do Rio Cubatão durante o período total do monitoramento foi de apenas $2,54 \%$, e de $2,9 \%$ para a cota mínima. Por sua vez, a carga hidráulica máxima do poço PMN-02A apresentou redução de $12,6 \%$, após a paralisação do sistema de remediação e de 33,6\% de aumento na carga mínima. O poço PMN-04A apresentou variações de 9,2\% e 7\% de aumento em suas cargas máxima e mínima, respectivamente

As variações dos valores e amplitude das cargas hidráulicas nos poços de monitoramento tem influência direta nos gradientes hi dráulicos (i) do aquífero na região de contato com o Rio Cubatão.

As figuras 5, 6 e 7 apresentam as vari- 
ações dos gradientes hidráulicos entre os poços de monitoramento PMN-02A e PMN-04A, PMN-02B e PMN-04B, e PMN-02C, PMN04B. Em função das constantes oscilações de nível do aquífero em resposta à variação de carga do Rio Cubatão, os gradientes hidráulicos também variam de maneira constante.

A Figura 5 apresenta as oscilações do gradiente hidráulico entre os poços PMN-02A e PMN-04A, na porção superior do aquífero livre (camadas 1 e 2). O sinal negativo do gradiente hidráulico indica que o fluxo do aqui- fero se dá predominantemente no sentido do Rio Cubatão para o aquífero da área de estudo na porção superior do aquífero livre. Tal verificação corrobora com a afirmação de Gasparini (2014) de que o Rio Cubatão é influente na área de estudo. Os picos positivos de variação gradientes hidráulicos (ou maiores diferenças entre gradientes hidráulicos) ocorrem quando o sistema de remediação está ativo, aumentando a variação dos gradientes hidráulicos neste período.

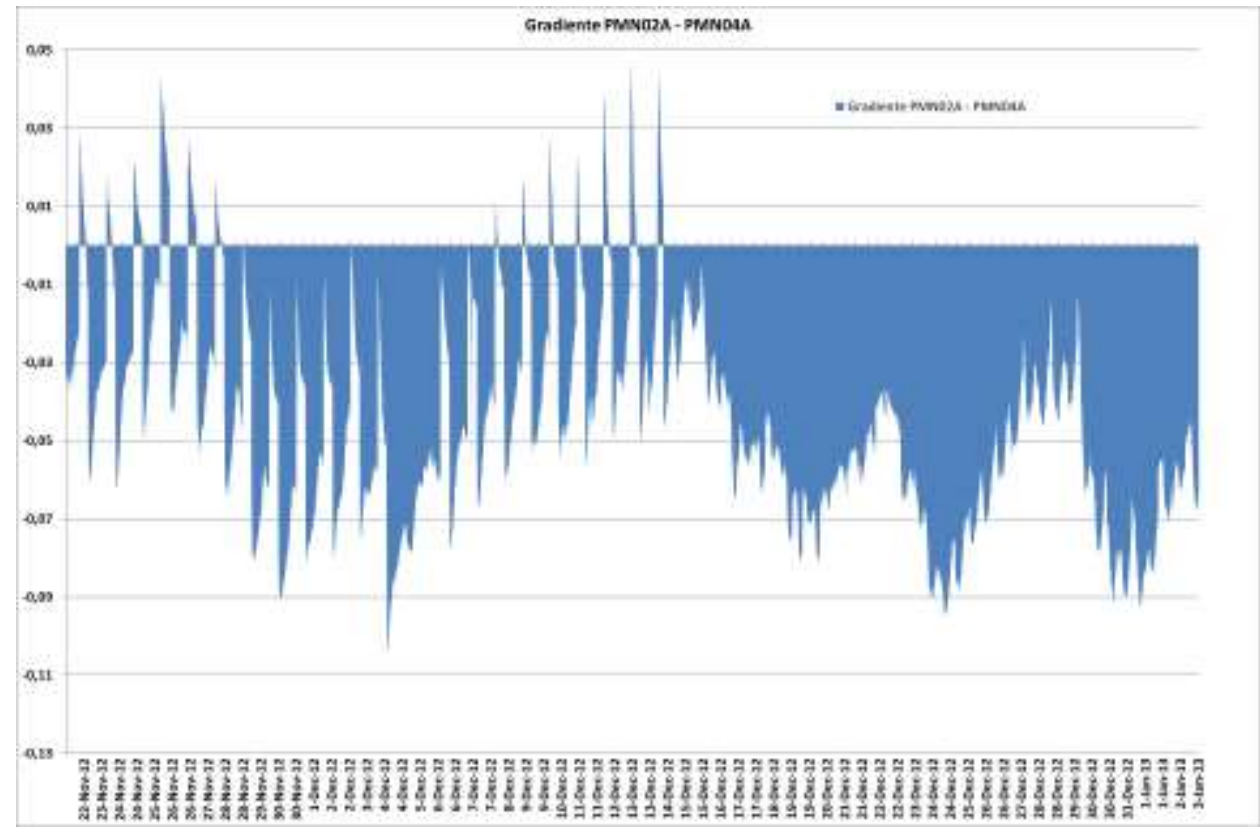

Figura 5 - Variações dos gradientes hidráulicos entre os poços PMN-02A, PMN-04A (22/11 a 03/01/2013)

Figure 5 - Hydraulic gradient fluctuations between monitoring wells PMN-02A, PMN-04A (Nov $22^{\text {nd }} 2012$ to Jan $3^{\text {rd }} 2013$ )

O comportamento de gradientes hidráulicos entre os poços PMN-02B e PMN04B (Figura 6), posicionados na porção intermediária do aquífero livre, apresentam oscilações de magnitude positiva e negativa. No período no qual o sistema de remediação permaneceu desligado em manutenção, a amplitude dos gradientes foi substancialmente reduzida. Os gradientes com valores positivos indicam que o fluxo, na porção intermediária do aqui- fero, dá-se do aquífero para o Rio Cubatão, descarregando no rio. No entanto, os gradientes apresentam oscilação contínua de magnitude e direção. No período em que os gradientes apresentam valores negativos, o fluxo do aquífero dá-se no sentido do Rio Cubatão para o aquífero, recarregando-o. Portanto, na porção intermediária do aquífero, o sentido de fluxo do aquífero apresenta comportamento de carga e descarga (efluência e influência). 


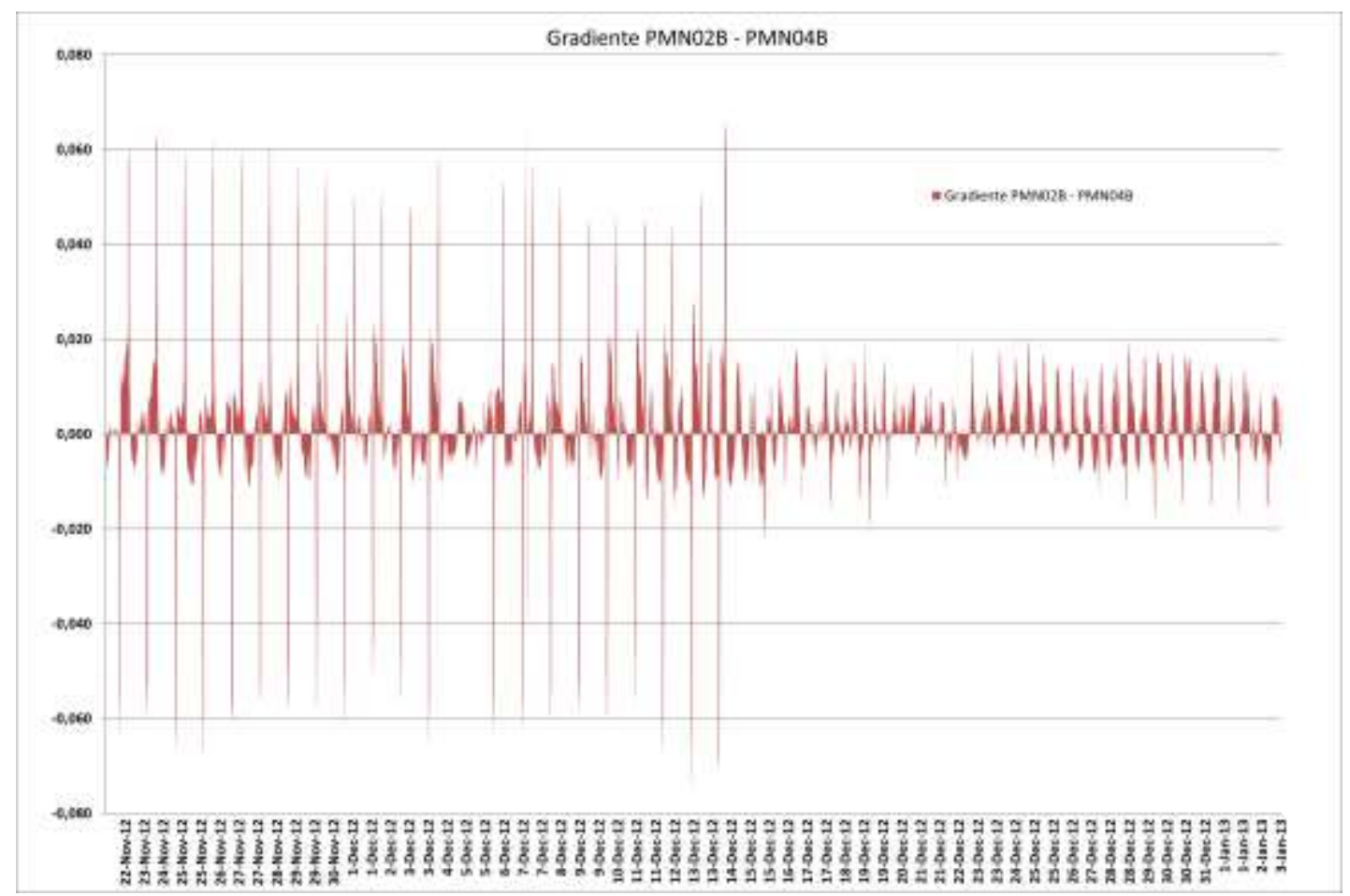

Figura 6 - Variações dos gradientes hidráulicos entre os poços PMN-02B, PMN-04B (22/11/2012 a 03/01/2013)

Figure 6 - Hydraulic gradient fluctuations between monitoring wells PMN-02B, PMN-04B (Nov $22^{\text {nd }}$ 2012 to Jan $3^{\text {rd } 2013)}$

Nos poços PMN-02C e PMN-04B, porção profunda do aquífero livre, conforme a Figura 7, o comportamento dos gradientes é similar ao constatado na porção intermediária (poços PMN-02B e PMN-04B). Nesta porção inferior, o fluxo do aquífero livre também apresenta ciclos intercalados de gradientes po- sitivos e negativos, indicando momentos de descarga no Rio Cubatão e recarga do aquífero pelo rio. Por motivo de término da bateria interna, o transdutor de pressão instalado no poço $\mathrm{PMN}-02 \mathrm{C}$ registrou as cargas hidráulicas somente até às 4:40 da manhã do dia 20 de dezembro de 2012. 


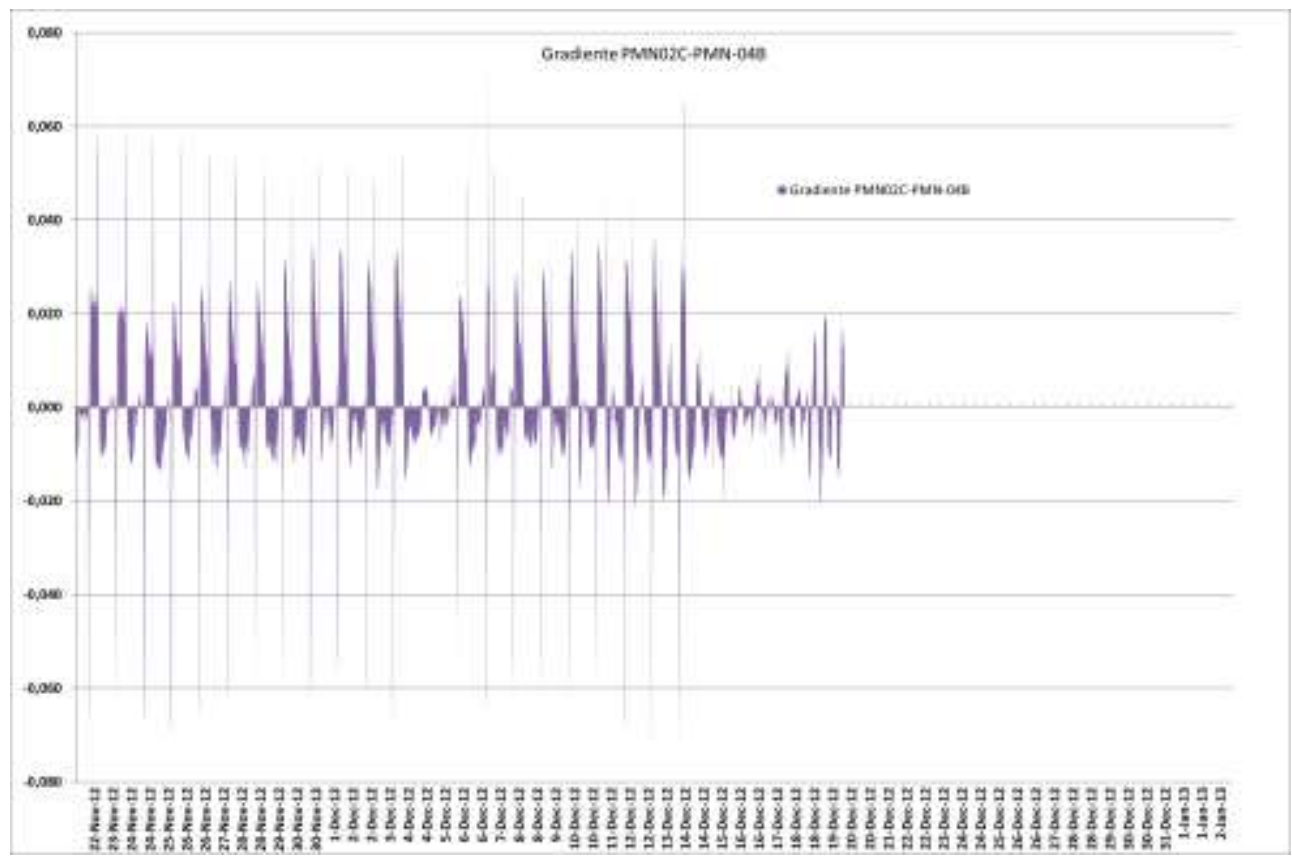

Figura 7 - Variações dos gradientes hidráulicos entre os poços PMN-02C, PMN-04B (22/11/2012 a 20/12/2012)

Figure 7 - Hydraulic gradient fluctuations between monitoring wells PMN-02C, PMN-04B (Nov $22^{\text {nd }}$ to Dec 20 ${ }^{\text {th }}$ 2012)

\subsection{Aplicação do Método das Médias Móveis (Serfes 1991)}

A carga hidráulica média do Rio $\mathrm{Cu}-$ batão, PMN-02A, PMN-02B, PMN-02C, PMN-04A, PMN-04B, OW-24B e OW-24C foi calculada com os resultados da utilização do método de Serfes (1991) na série de dados de 22 de novembro a 14 de dezembro de 2012, quando o aquífero respondeu às oscilações do Rio Cubatão e ao regime de operação do sistema de remediação (Tabela 3).

Tabela 3 - Cargas Hidráulicas Médias pelo Método das Médias Móveis (Serfes 1991) no período de 22/11 a 14/12/2012 (sistema de remediação ativo)

Table 3 - Mean hydraulic heads by Moving Average Method (Serfes 1991) in the period from Nov $22^{\text {nd }}$ to Dec $14^{\text {th }} 2012$ (active remediation system)

\begin{tabular}{ccccccccc}
\hline & Rio & PMN- & PMN- & PMN- & PMN- & PMN- & OW- & OW- \\
Cubatão & 02A & 02B & 02C & 04A & 04B & 24B & $24 \mathrm{C}$ \\
\hline $\begin{array}{c}\text { Carga } \\
\text { Hidráulica } \\
\text { Média } \\
(\mathrm{m})\end{array}$ & 0,968 & 1,449 & 1,449 & 1,454 & 2,106 & 1,417 & 2,304 & 1,795 \\
\hline
\end{tabular}

Com base nas cargas hidráulicas médias (Tabela 3) dos pontos monitorados, calculou-se então os gradientes hidráulicos médios para o aquífero afetado pela influência da maré em ambiente estuarino pela equação:

$$
\bar{\imath}=\frac{M_{1}-M_{2}}{l}
$$

(Equação 2) $\bar{\imath}=$ gradiente hidráulico médio $(\mathrm{L} / \mathrm{L})$

$\mathrm{M}_{1}=$ Carga hidráulica média no ponto 1 (L)

$\mathrm{M}_{2}=$ Carga hidráulica média no ponto 2 (L)

$l=$ distância entre o ponto 1 e $2(\mathrm{~L})$

A distância entre os poços PMN02A, PMN2B, PMN-02C, localizados no interior da área de estudo, e os poços PMN-04A e PMN-04B, no talude da margem esquerda do Rio Cuba-

onde: 
tão é de $12,70 \mathrm{~m}$. Aplicando as cargas hidráulicas médias listadas na Tabela 4 e a distância de 12,70 na Equação 2, os gradientes hidráulicos médios são obtidos no eixo definido pelo alinhamento dos conjuntos de poços PMN-02 e PMN-04.

Os gradientes hidráulicos médios, ob- tidos durante o sistema de remediação ativo (Tabela 4), indica influência do rio Cubatão nos níveis mais rasos do aquífero livre (entre PMN02A e PMN04A, nas camadas 1 a 2), ou seja, sentido de fluxo predominante das águas subterrâneas do rio para aquífero, e no nível profundo, sentido de fluxo inverso (fluxo de água subterrânea descarregando no rio).

Tabela 4 - Gradientes Hidráulicos Médios no período de 22/11 a 14/12/2012 (sistema de remediação ativo)

Table 4 - Mean hydraulic gradients in the period from Nov $22^{\text {nd }}$ to Dec $20^{\text {th }} 2012$ (active remediation system)

\begin{tabular}{lccc}
\hline & PMN02A-PMN04A & PMN02B-PMN04B & PMN02C-PMN04B \\
\hline $\begin{array}{l}(\bar{l}) \text { Gradiente Hidráulico } \\
\text { Médio }(\mathrm{m} / \mathrm{m})\end{array}$ & $-0,0517$ & 0,0026 & 0,0030 \\
\hline
\end{tabular}

As cargas hidráulicas médias e os gradientes médios no período em que o sistema de remediação encontrava-se em manutenção preventiva, estão apresentados nas tabelas 5 e 6 , respectivamente.

Tabela 5 - Cargas Hidráulicas Médias pelo Método das Médias Móveis (Serfes 1991) no período de 14/12/2012 a 03/01/2013 (sistema de remediação inativo)

Table 5 - Mean hydraulic heads by Moving Average Method (Serfes 1991) in the period from Dec $14^{\text {th }} 2012$ to Jan $3^{\text {rd }} 2013$ (inactive remediation system)

\begin{tabular}{|c|c|c|c|c|c|c|}
\hline & $\begin{array}{c}\text { Rio } \\
\text { Cubatão }\end{array}$ & PMN-02A & PMN-02B & PMN-02C & PMN-04A & PMN-04B \\
\hline $\begin{array}{c}\text { Carga } \\
\text { Hidráulica } \\
\text { Média } \\
\text { (m) }\end{array}$ & 1,160 & 1,541 & 1,601 & 1,601 & 2,081 & 1,575 \\
\hline
\end{tabular}

Tabela 6 - Gradientes Hidráulicos Médios no período de 14/12/2012 a 03/01/2013, calculados com médias móveis de carga hidráulica (sistema de remediação desativado)

Table 6 - Mean hydraulic gradients $n$ the period from Dec $14^{\text {th }} 2012$ to Jan $3^{\text {rd }} 2013$ (inactive remediation system) PMN02A-PMN04A $\quad$ PMN02B-PMN04B PMN02C-PMN04B
( $\bar{l}$ ) Gradiente Hidráulico
Médio $(\mathrm{m} / \mathrm{m})$
$-0,0425$
$-0,0027$
0,0020

Com o sistema de remediação desativado (Tabela 6), os gradientes hidráulicos médios resultantes indicaram influência do rio nos níveis mais rasos (nas camadas 1 e 2), e efluência na Camada 3. Estes comportamentos predominantes podem ser visualizados nas figuras 5, 6 e 7. Os gradientes hidráulicos entre os poços PMN-02A e PMN-04A há o predomínio dos gradientes negativos nos dois cenários. Nos gradientes hidráulicos entre os poços PMN-02B e PMN-04B há diferenciação entre os valores de gradientes hidráulicos (positivos e negativos) durante e após a paralisação do sistema de remediação. No entanto, as Águas Subterrâneas (2016) 30(3):427-439. frequências de ocorrência dos valores positivos (no cenário $\mathrm{A}$, sistema de remediação ativo) são destacadas em relação aos negativos (cenário $\mathrm{B}$, sistema de remediação inativo). O mesmo ocorre no nível profundo (Camada 3); no entanto apesar da maior frequência dos valores negativos, os valores de gradiente hidráulico positivos são maiores resultando em gradiente hidráulico médio positivo.

Comparando-se a média aritmética de todas as medições realizadas de 22 de novembro a 14 de dezembro de 2012, quando as cargas hidráulicas responderam às oscilações da maré e pressões introduzidas pelo sistema de 
remediação, com as cargas hidráulicas médias calculadas pelo Método das Médias Móveis (SERFES 1991), verificam-se diferenças normatizadas na faixa de 4 a $11,1 \%$ para a cargas hidráulicas nos pontos monitorados, conforme Tabela 7. Nos poços mais distantes do sistema de remediação (OW-24A e OW24-B) essas diferenças foram menores $(4,0 \%$ e $5,9 \%)$ em relação aos poços mais próximos ao rio e a esse sistema (PMN-02A, PMN-02B, PMN02C e PMN-04A e PMN-04B).

Tabela 7 - Comparativo da Média Aritmética das Cargas Hidráulicas do Período Total de Monitoramento e da Média Móvel pelo Método de Serfes (1991) num período de 72 horas sob influência das variações da maré e sistema de remediação ativo

Table 7 - Comparative of hydraulic heads arithmetic mean of monitoring total period and the moving average method of Serfes (1991) for 72 hours under the influence of tide fluctuation and active remediation system

\begin{tabular}{lcccccccc}
\hline $\begin{array}{c}\text { 22/nov - } \\
\text { 20/dez }\end{array}$ & $\begin{array}{c}\text { Rio } \\
\text { Cubatão }\end{array}$ & PMN-02A & PMN-02B & PMN-02C & PMN-04A & PMN-04B & 24A & OW-24B \\
\hline Média & & & & & & & & \\
Aritmética & 1,089 & 1,552 & 1,551 & 1,555 & 2,310 & 1,538 & 2,399 & 1,908 \\
Média Serfes & & & & & & & & \\
$(1991)$ & 0,968 & 1,449 & 1,449 & 1,454 & 2,106 & 1,417 & 2,304 & 1,795 \\
Diferença \% & $11,1 \%$ & $6,6 \%$ & $6,6 \%$ & $6,5 \%$ & $8,8 \%$ & $7,9 \%$ & $4,0 \%$ & $5,9 \%$ \\
\hline
\end{tabular}

Da mesma maneira, verificam-se diferenças normatizadas de $-3,4$ a $13,4 \%$ no período de 14 de dezembro de 2012 a 3 de janeiro de 2013, apresentadas na tabela 8, quando o rio e o aquífero responderam apenas às oscila- ções da maré. Os poços mais distantes (OW24A e OW24-B) do rio apresentaram as menores diferenças em relação aos demais poços (PMN-02A, PMN-02B, PMN-02C e PMN04A e PMN-04B).

Tabela 8 - Comparativo da Média Aritmética das Cargas Hidráulicas do Período Total de Monitoramento e das Média Móvel pelo Método de Serfes (1991) num período de 72 horas apenas sob influência das variações da maré (sistema de remediação desativado)

Table 8 - Comparative of hydraulic heads arithmetic mean of monitoring total period and the moving average method of Serfes (1991) for 72 hours under the influence of tide fluctuation and inative remediation system

\begin{tabular}{|c|c|c|c|c|c|c|c|c|}
\hline 21/dez - 3/jan & $\begin{array}{c}\text { Rio } \\
\text { Cubatão }\end{array}$ & PMN-02A & PMN-02B & PMN-02C & PMN-04A & PMN-04B & OW-24A & OW-24B \\
\hline Média & & & & & & & & \\
\hline Aritmética & 1,097 & 1,683 & 1,697 & 1,711 & 2,403 & 1,679 & 2,54 & 2,049 \\
\hline $\begin{array}{l}\text { Média Serfes } \\
\text { (1991) }\end{array}$ & 1,160 & 1,541 & 1,601 & 1,601 & 2,081 & 1,575 & 2,634 & 2,118 \\
\hline Diferença \% & $-5,7 \%$ & $8,4 \%$ & $5,7 \%$ & $6,4 \%$ & $13,4 \%$ & $6,2 \%$ & $-3,7 \%$ & $-3,4 \%$ \\
\hline
\end{tabular}

\section{CONCLUSÕES}

O método das médias móveis pode ser utilizado quando o estudo busca avaliar o sentido predominante do fluxo das águas subterrâneas em sistemas dinâmicos como o estuário e regiões costeiras, pois permite a obtenção das cargas potenciométricas e gradientes médios por meio do monitoramento contínuo das cargas hidráulicas de 72 horas, com coleta de dados de nível a cada 1 hora.

Neste estudo, a diferença máxima entre a média aritmética das cargas hidráulicas (coletadas em intervalos de 10 minutos, por um período de 42 dias), e os valores médios obtidos pelo Método das Médias Móveis com dados de nível a cada 1 hora pelo período de 72 horas consecutivas, foi de $13,4 \%$. 
Dada a complexidade do comportamento hidráulico do ambiente estuarino monitorado em função das oscilações diárias da maré, precipitações, operação de um sistema de remediação que introduz pressões no aquífero e alterações antrópicas na vazão do rio, o

\section{REFERÊNCIAS}

ABARCA, E.; KARAM, H.; HEMOND, H.F.; HARVEY, C.F. Transient groundwater dynamics in a coastal aquifer: The effects of tides, the lunar cycle, and the beach profile. Water Resources Research, v.49, 2473-2488, doi:10.1002/wrcr.20075, 2013.

AECOM. Construção e Montagem dos Poços do Sistema de Air Sparging - Módulo 3. São Paulo: AECOM. 2012. 307p.

BATISTA FILHO, J.J. A dinâmica das águas subterrâneas no estuário do rio Itanhaém, litoral sul do estado de São Paulo. 2006. Dissertação (Mestrado em Geociências) - Universidade Estadual de Campinas.

CUBATÃO PREFEITURA MUNICIPAL. Aspectos Geográficos. Disponível em: <http://www.Cubatão.sp.gov.br/historia/cidade/aspectos-geograficos/> Acesso em: 15 mar 2015.

GASPARINI, F.B., 2014. Análise da Influência de Parâmetros Ambientais e Antrópicos Sobre a Dinâmica de Aquíferos Estuarinos: Estudo de Caso em Área Industrial, Cubatão, SP. 2014. Dissertação (Mestrado em Geociências) - Universidade Estadual de Campinas.

RICARDI-BRANCO, F.; YOSHINAGA, S.; CARDINALE, F.; PEREIRA, P.R.B. Accumula-
Método das Médias Móveis leva em consideração as oscilações harmônicas produzidas de modo que os resultados sejam valores confiáveis e bastante aceitáveis, em detrimento de apenas uma média aritmética, dada a constante dinâmica do ambiente estudado.

tion of Bio Debris and Its Relation with the Underwater Environment in the Estuary of Itanhaem River, Sao Paulo State. In: Imran Ahmand Dar; Mithas Ahmad Dar. (Org.). Earth and Environmental Sciences. 1 ed. Rijeka: In Tech Publisher, 2011, v.2, p. 565-590.

ROBINSON, M.; GALLAGER, D.; REAY, W. Field observations of tidal and seasonal variations in ground water discharge to tidal estuarine surface water. Groundwater Monitoring and Remediation, v. 18, n. 1: 83-92. 1998.

SERFES, M. E. Determining the Mean Hydraulic Gradient of Ground Water Affected by Tidal Fluctuations. Groundwater, v. 29-4: 549-555.1991.

SOUZA, R.T.; ROSA FILHO, E.F.; SILVA, T.E.R.B.; HINDI, E.C.; MELO, H.C. Hidrogeologia Costeira: aspectos comparativos em áreas de sedimento recente do Quaternário Holoceno - litoral do Paraná. Bol. Paranaense de Geociências, v. 66-67: 13-22. 2012.

WESTBROOK, S.J., J.L. RAYNER, G.B. DAVIS, T.P. CLEMENT, P.L. BJERG, S.F. FISHER. Interaction Between Shallow Groundwater. Saline Surface Water and Contaminant Discharge at a Seasonally and Tidally Forced Estuarine Boundary. Journal of Hydrology 302 (255-269). 2005. 